\title{
The UTMOST: A hybrid digital signal processor transforms the Molonglo Observatory Synthesis Telescope.
}

M. Bailes ${ }^{1,2}$, A. Jameson ${ }^{1,2}$, C. Flynn ${ }^{1,2}$, T. Bateman ${ }^{3,4}$, E. D. Barr ${ }^{1,2,5}$, S. Bhandari ${ }^{1,2}$, J. D. Bunton ${ }^{4}$, M. Caleb $^{6,1,2}$, D. Campbell-Wilson ${ }^{3,2}$, W. Farah ${ }^{1}$, B. Gaensler ${ }^{3,2,9}$, A. J. Green ${ }^{3,2}$, R. W. Hunstead ${ }^{3}$, F. Jankowski ${ }^{1,2}$, E. F. Keane ${ }^{7,1,2}$, V. Venkatraman Krishnan ${ }^{1,2}$, Tara Murphy ${ }^{2,3}$, M. O'Neill ${ }^{1}$, S. Osłowski ${ }^{1}$, A. Parthasarathy ${ }^{1,2}$, V. Ravi ${ }^{1,2,8}$, P. Rosado ${ }^{1}$, D. Temby ${ }^{3}$

${ }^{1}$ Centre for Astrophysics and Supercomputing, Swinburne University of Technology, Mail H30, PO Box 218, VIC 3122, Australia

${ }^{2}$ ARC Centre of Excellence for All-Sky Astrophysics (CAASTRO),

${ }^{3}$ Sydney Institute for Astronomy, School of Physics A28, The University of Sydney, NSW 2006, Australia

${ }^{4}$ Australia Telecope National Facility, PO Box 76, Epping NSW 1710, Australia.

${ }^{5}$ Max-Planck-Institut für Radioastronomie (MPIfR), Auf dem Hügel 69, D-53121 Bonn, Germany

${ }^{6}$ Research School of Astronomy and Astrophysics, Australian National University, Cotter Road Weston Creek, ACT 2611, Australia

${ }^{7}$ SKA Organization, Jodrell Bank Observatory, Cheshire SK11 9DL, UK

${ }^{8}$ Cahill Center for Astronomy and Astrophysics, Caltech, Pasadena, CA 91125, USA

${ }^{9}$ Dunlap Institute for Astronomy and Astrophysics, The University of Toronto, Toronto, ON M5S 3H4, Canada

\begin{abstract}
The Molonglo Observatory Synthesis Telescope (MOST) is an 18,000 $\mathrm{m}^{2}$ radio telescope situated some 40 $\mathrm{km}$ from the city of Canberra, Australia. Its operating band $(820-850 \mathrm{MHz})$ is now partly allocated to mobile phone communications, making radio astronomy particularly challenging. In this paper we describe how the deployment of new digital receivers (RX boxes), Field Programmable Gate Array (FPGA) based filterbanks and server-class computers equipped with 43 GPUs (Graphics Processing Units) has helped transform MOST into a versatile new instrument (the UTMOST) for studying the dynamic radio sky on millisecond timescales, ideal for work on pulsars and Fast Radio Bursts (FRBs). The filterbanks, servers and their high-speed, lowlatency network form part of a hybrid solution to the observatory's signal processing requirements. The emphasis on software and commodity off-the-shelf hardware has enabled rapid deployment through the reuse of proven 'software backends' for much of its signal processing needs. The new RX boxes have ten times the bandwidth of the original MOST and double the sampling of the cylindrical reflector line feed, which doubles the field of view. The servers' record and playback capability has facilitated rapid debugging of many new observing modes. Inherent to the solution is the capacity to run commensal science operations. The UTMOST can simultaneously excise interference, make maps, coherently dedisperse pulsars, and perform real-time searches of coherent fan beams for dispersed single pulses. Although system performance is still sub-optimal, a pulsar timing and FRB search programme has commenced and the first UTMOST maps have been made. The telescope operates as a robotic facility, deciding how to efficiently target pulsars and how long to stay on source, via feedback from real-time pulsar folding. The regular timing of over 300 pulsars has resulted in the discovery of 7 pulsar glitches and 3 FRBs. The UTMOST demonstrates that if sufficient signal processing can be applied to the voltage streams it is possible to perform innovative radio science in hostile radio frequency environments.
\end{abstract}

Keywords: techniques: interferometric instrumentation: interferometers stars: pulsars: general

\section{Introduction}

The UTMOST ${ }^{1}$ is a major upgrade of the Molonglo Observatory Synthesis Telescope (MOST), which consists

$1{ }^{1}$ UTMOST is not an acronym 
of two EW-aligned cylindrical paraboloid reflectors with a collecting area of $18,000 \mathrm{~m}^{2}$, situated in a valley 40 km east of Australia's capital city, Canberra. The length of each cylindrical arm is $778 \mathrm{~m}$, separated by a $15-\mathrm{m}$ gap. It is a synthesis interferometer which was originally part of the transit instrument known as the Mills Cross (Robertson 1991), and is sensitive to right-hand (IEEE) circularly polarised radiation only. The telescope played a pivotal role in the early years of radio pulsar astronomy due to its large collecting area and compact design. Discoveries included the first association of a pulsar (in Vela) with a supernova remnant (Large et al. 1968) and 155 new pulsars from the prolific second Molonglo pulsar survey (Manchester et al. 1978).

The MOST re-used some of the electronics and analogue systems from the Molonglo Cross and became a synthesis imaging facility. It processed a single $3-\mathrm{MHz}$ channel of data streamed from each of 88 18.29-m elements of the telescope, operating at a central frequency of $843 \mathrm{MHz}$. Images were produced by the back projection of fan beams formed in real time using an analogue, hard-wired beamformer.

As a synthesis array, the MOST produced many important surveys and catalogues, including the Sydney University Molonglo Sky Survey (SUMSS; Bock et al. 1999b; Mauch et al. 2003), two Galactic Plane Surveys, MGPS-1 (Green et al. 1999) and MGPS-2 (Murphy et al. 2007; Green et al. 2014), the MOST Supernova Remnant Catalogue (MSC; Whiteoak \& Green 1996), and the Molonglo Southern 4 Jy Survey (Burgess \& Hunstead 2006) (the Southern counterpart to the Northern 3CR catalog). It was particularly useful for rapid follow-up observations of transient sources. For example, it detected the prompt radio emission from supernova SN1987A (Turtle et al. 1987) and discovered radio emission from the micro-quasar GRO 1655-40 (della Valle et al. 1994). A systematic monitoring campaign of SN 1987A led to its re-detection as a radio supernova remnant (Staveley-Smith et al. 1992).

\subsection{The SKAMP Upgrades}

In 2004, staged upgrades were proposed for the MOST as part of the "Square Kilometre Array Molonglo Pathfinder" (SKAMP) project, to give the instrument its third major lease of scientific life. The first upgrade, SKAMP-1 (Adams et al. 2004), replaced the analogue beamformer with an 8-bit digital correlator while retaining the same bandwidth and field of view. The second upgrade, SKAMP-2 (de Souza et al. 2007), increased the system bandwidth from 3 to $30 \mathrm{MHz}$ and the instantaneous field of view by a factor of two (by processing all 352 radio frequency outputs from the 352 independent outputs of the telescope rather than precombining them into 176 pairs). This involved laying over $200 \mathrm{~km}$ of optical fibre to 88 new 4-input digital receivers (RX boxes) in the field. Each RX box received its inputs from one 18-m element and the digital optical transmission from the RX boxes replaced the analogue coax cable used in MOST. Transmission of clocks to the RX boxes was also upgraded to optical fibre. The SKAMP-2 optical fibre outputs (from the 88 RX boxes) feed 22 polyphase filterbanks (PFBs) in the main control room. Each PFB is built on an ATCA board using Xilinx Virtex 4 FPGAs (Field Programmable Gate Arrays).

In late 2013, a new system design (the UTMOST) was proposed that could add greater flexibility to the signal processing chain by using software and commodity offthe-shelf servers and graphics processing units instead of the Field Programmable Gate Arrays proposed for use in SKAMP-2. Signals to the GPUs would be provided by the MOST cylindrical reflector and feed, and the SKAMP-2 RX boxes and FPGA filterbanks.

\subsection{Radio Astronomy in Software}

An emerging trend in radio astronomy over the past decade is the replacement of custom chips with commodity, off-the-shelf (COTS) signal processors such as Graphics Processing Units (GPUs) and software. While less energy efficient than correlators using Application Specific Integrated Circuits (ASICs) or Field Programmable Gate Arrays (FPGAs), software correlators and signal processors are particularly inexpensive to develop. Changes to the configuration and operational modes are easy to implement after construction, as this is predominantly achieved in software. Examples of software correlators (or "coherent dedispersers") currently in use for pulsar observations are CPSR2 (Bailes 2009) and GUPPI (DuPlain et al. 2008). Further, it has become straightforward to publish and distribute software instruments such as the pulsar processor DSPSR (van Straten \& Bailes 2011) via online repositories, for installation by other users. Other examples of software baseband processing instruments are the VLBI correlator DiFX (Deller et al. 2007) and XGPU (Clark et al. 2012) a GPU-based correlator for "large-N" interferometers such as the Murchison Widefield Array (MWA) (Tingay et al. 2013) and the Large-Aperture Experiment to Detect the Dark Ages (LEDA) (Greenhill et al. 2014).

A software backend for the MOST/SKAMP was proposed in early 2013 , when its potential to become a powerful Fast Radio Burst discovery engine working commensally with other observing modes was realised. The UTMOST retains the digital receivers and polyphase filterbanks of the original SKAMP-2 system, but performs the rest of the signal processing on commodity CPUs and GPUs. The UTMOST Software Correlator has been designed to provide great processing flexibility and has commensal modes that enable simultaneous 
Table 1 UTMOST telescope parameters.

\begin{tabular}{l|c}
\hline Collecting Area & $18000 \mathrm{~m}^{2}$ \\
Number of RF inputs & 352 \\
Operating band & $820-850 \mathrm{MHz}$ \\
Primary beam dimensions & $4.25 \mathrm{deg} \times 2.8 \mathrm{deg}$ \\
Fan beam dimensions & $46 \operatorname{arcsec} \times 2.8 \mathrm{deg}$ \\
Theoretical gain & $3.5 \mathrm{~K} \mathrm{Jy}^{-1}$ \\
Effective gain (Nov 2016) & $\sim 0.5 \mathrm{~K} \mathrm{Jy}^{-1}$ \\
SEFD (typical) & $115 \mathrm{Jy}$ \\
$T_{\text {sys }}$ (best inputs) & $100 \mathrm{~K}$ \\
\hline
\end{tabular}

mapping, pulsar coherent dedispersion, burst-searching and baseband dump modes whilst employing novel interference mitigation strategies.

This paper describes the scientific capability and design of the UTMOST, the Swinburne University of Technology CPU/GPU backend solution for the MOST. In $\S 2$ we provide a brief description of all system components and their technical specifications. The site is subject to interference from domestic mobile phones and communications towers, and a simple technique to detect and attenuate their signals is described in §3. Examples of the major observing modes are provided in $\S 4$, the initial science programs are described in $\S 5$, and our summary and future prospects are given in $\S 6$.

\section{UTMOST System Design}

The MOST consists of two parabolic cylinders $11.6 \mathrm{~m}$ wide and $778 \mathrm{~m}$ long separated by a $15-\mathrm{m}$ gap. Parabolic cylinders enable a linear scaling of cost with collecting area but make the steering of the beam along its axis rather complex. The MOST uses 352 modules each comprising 22 ring antennas of circumference $\lambda(36 \mathrm{~cm})$ for operation at the central frequency of the $843 \mathrm{MHz}$ feed. These ring antennas are coherently summed in resonant cavities along the focal line of the paraboloid, before being fed to 352 Low Noise Amplifiers (LNAs) (Robertson 1991). The signals from each LNA are filtered and down-converted before being 8-bit complex sampled in the field at 100 Megasamples/sec (i.e $200 \times 10^{6} \mathrm{~B} / \mathrm{s}$ ). This is equivalent to Nyquist sampling a bandwidth of $100 \mathrm{MHz}$ at a rate of $200 \mathrm{Msamples} / \mathrm{sec}$. They are transmitted via multicore multi-mode fibre to digital Polyphase Filterbanks (PFBs) in a central building $100 \mathrm{~m}$ north of the junction between the two arms of the telescope. Here the data stream is processed by the UTMOST software correlator backend. A block diagram of the system is shown in Figure 1 and the basic telescope parameters provided in Tables 1 and 2. Each of these stages is now described in more detail.

\subsection{The Front-end Feeds}

In order to steer the primary beam, each telescope section in the East-West direction uses an ingenious system of resonant ring antennas which are sensitive to righthand (IEEE) circularly polarised radiation. The ring antennas are differentially rotated to steer the phasedarray beam and coherently add incident radiation in groups of 22 antennas spanning $4.57 \mathrm{~m}$ of telescope length, producing 352 independent outputs called modules (176 on each arm). In the North-South direction steering is comparatively simple, as each telescope arm can be mechanically tilted from the zenith, enabling observation of all parts of the sky south of declination $\delta=+17^{\circ}$.

The gain of each module is approximately $G=0.01 \mathrm{~K}$ $\mathrm{Jy}^{-1}$, where we include an effective aperture factor $\eta=0.5$. The system temperature in the best performing modules is estimated, from observations of point source calibrators, to be $T_{\text {sys }} \sim 100 \mathrm{~K}$. The ratio $G / T_{\text {sys }}$ presently can vary considerably (by up to an order of magnitude) from module to module, as legacy sources of self-generated RFI are still being identified and removed in the ongoing overhaul of the entire system. Currently, in a typical observation, the best modules achieve a system equivalent flux density (SEFD) of $\sim 10 \mathrm{kJy}$, while a typical performance figure for the whole instrument is SEFD $=110 \mathrm{Jy}$, due to the combination of many factors, including incorrect phasing, ring antenna misalignment, self-induced RFI and cross-talk, RX box performance, and deformations in the telescope structure. Despite this range in performance, substantial verification science has been carried out during the upgrade (see §5).

The LNAs contribute $\sim 20 \mathrm{~K}$ (as measured in the laboratory) to the total system temperature, with the remainder coming from a variety of sources including mesh leakage, spillover and cable feeds. The effective gain of each module varies in a complex manner as it is steered East-West, a function termed the "meridian distance gain curve" (Gaensler \& Hunstead 2000). This arises endemically in parabolic antennas because of multiple reflections along the cylinder which attenuate the signal to give an effective gain of $<0.01 \mathrm{~K} \mathrm{Jy}^{-1}$ per module. When added coherently on the meridian, the 352 modules of the antenna should combine to produce a gain of $3.5 \mathrm{~K} \mathrm{Jy}^{-1}$ and an SEFD of $\sim 30 \mathrm{Jy}$.

Four adjacent modules feed the independent RF signals from their LNAs via coaxial cable to one of 88 RX boxes. Prior to the RX boxes, the UTMOST signal chain is identical to the MOST. The MOST operated by coherently combining adjacent modules, whereas SKAMP-2 retains the independent inputs from each group of 22 ring antennas and brings back the digitized signals on optical fibre. 

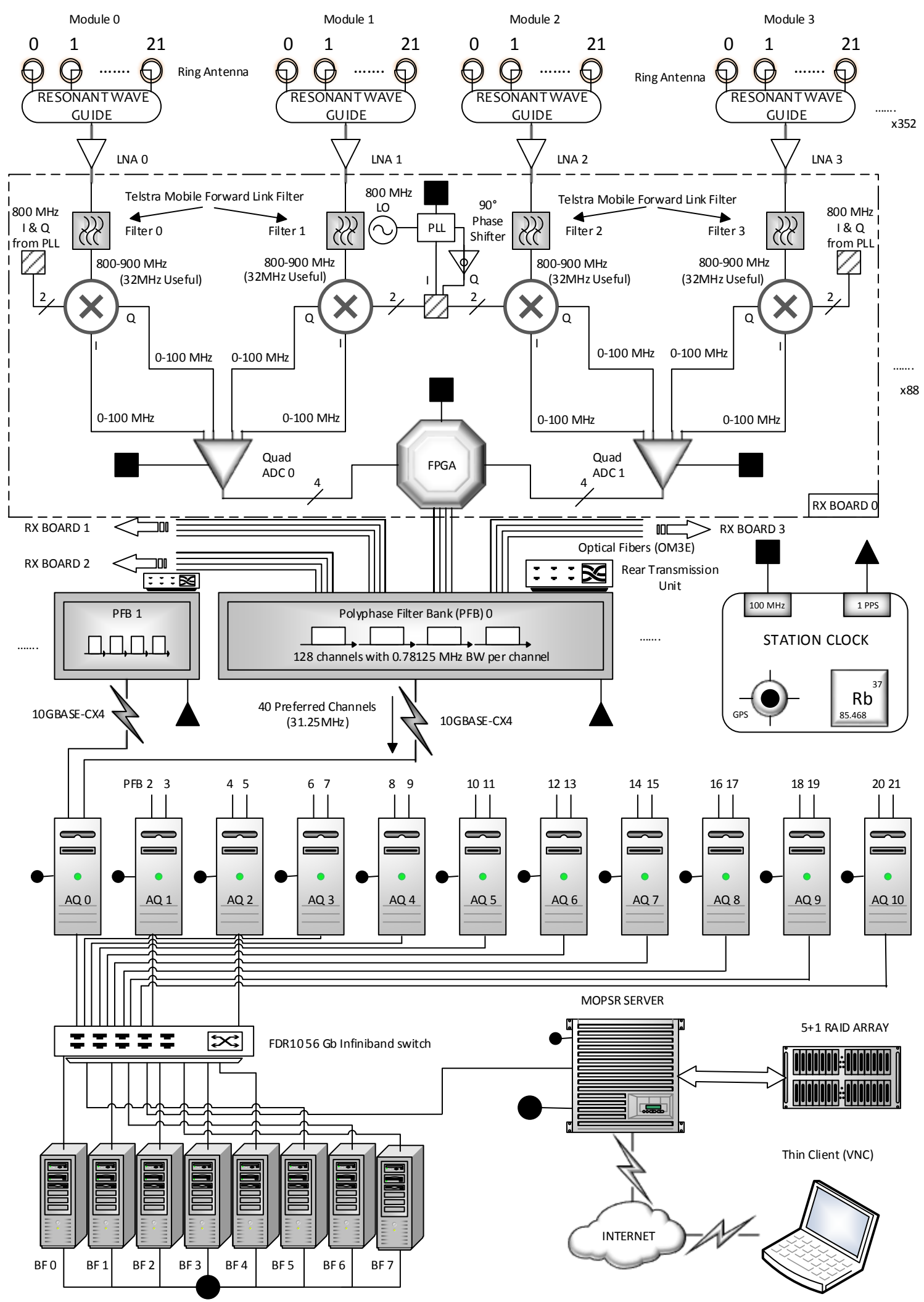

Figure 1. The UTMOST block diagram. From the ring antennas to the LNAs the system is identical to that used on the MOST. The SKAMP-2 upgrade includes the RX boxes and the polyphase filterbank (PFB), at which point the UTMOST backend taps off data via $10 \mathrm{~Gb}$ UDP packets using the CX-4 ports on the PFBs. The UTMOST uses commodity off-the-shelf computing hardware (marked "AQ" and "BF" nodes in the figure) and highly flexible software algorithms to achieve its signal processing requirements. AQ denotes the system's "data acquisition" nodes and BF the system's "beam forming" nodes. 
Table 2 Frequency resolution, time resolution, number of fan beams and coherent dedispersion properties for the UTMOST's operational modes.

\begin{tabular}{l|lc}
\hline Single Module Mode & Processed bandwidth & $31.25 \mathrm{MHz}$ \\
& Number of frequency channels & 128 \\
& Time resolution & $655.36 \mu \mathrm{s}$ \\
& Output data rate & $0.19 \mathrm{MB} / \mathrm{sec} / \mathrm{modul}$ \\
\hline LORES Fan Beam Mode & Number of frequency channels & 128 \\
& Time resolution & $655.36 \mu \mathrm{s}$ \\
& Simultaneous pulsar coherent dedispersion & Yes \\
& Number of fan beams & 352 \\
& Fan beam dimensions (FWHP) & $46 \mathrm{arcsec} \times 2.8 \mathrm{deg}$ \\
& Output data rate & $0.19 \mathrm{MB} / \mathrm{sec} / \mathrm{modul}$ \\
\hline \multirow{2}{*}{ HIRES Fan Beam Mode } & Number of frequency channels & 1024 \\
& Time resolution & $327.68 \mu \mathrm{s}$ \\
& Simultaneous pulsar coherent dedispersion & Yes \\
& Number of fan beams & 704 \\
& Fan beam dimensions (FWHP) & $46 \mathrm{arcsec} \times 2.8 \mathrm{deg}$ \\
& Output data rate & $1.56 \mathrm{MB} / \mathrm{sec} / \mathrm{module}$ \\
\hline FX Calibration Mode & Number of frequency channels & 1024 \\
& Number of baseline products & $8 \times 351=2808$ \\
& Simultaneous pulsar coherent dedispersion & $\mathrm{No}$ \\
\hline FX Correlator Mode & Number of frequency channels & 128 \\
& Number of baseline products & $352 \times 351 / 2=61776$ \\
& Simultaneous pulsar coherent dedispersion & $\mathrm{No}$ \\
\hline
\end{tabular}

\subsection{The Digital Receiver RX boxes and 3G Filters}

The 88 RX boxes apply a pair of commercially sourced analogue filters to each of the four inputs in series to filter out local telecommunications $3 \mathrm{G}$ and $4 \mathrm{G}$ up-links and produce four band-limited signals, currently from 820 to $850 \mathrm{MHz}$. These are down-converted with an $850 \mathrm{MHz}$ local oscillator, and the complex signals are transmitted via four optical fibres using SerDes format (achieved on a Lattice FPGA) to the central processing building.

\subsection{The Polyphase Filterbanks}

Optical fibres carrying the digitised signal from sets of $4 \mathrm{RX}$ boxes at a time (i.e. 16 inputs) are aggregated in the central processing building in a Rear Transmission Module (RTM), which transmits the data to polyphase filterbanks (PFBs). The PFBs have a CX-4 port, which is used to send 8k UDP ethernet packets to industry standard (copper) 10 Gb network interface cards (NICs). The PFBs use the SKAMP-2 hardware with firmware modified to bypass the cross connect, "cornerturn" and fine filterbank operation. Only the 128 coarse filterbanks remain, providing data with a 781-kHz channel spacing. The original SKAMP-2 filterbanks were oversampled by a factor of $32 / 27$, to reduce signal loss at the coarse channel edges. Two extra modes are imple- mented for UTMOST: a critically sampled 128-channel filterbank and a pass-through mode. Selection of the mode is programmable. The pass-through mode proved pivotal in debugging the more complex modes during system implementation, but the default run mode is the critically-sampled one. The over-sampled mode is reserved for future use, and may help eliminate loss of sensitivity between frequency channels, at the cost of a slightly increased data rate. The filterbank modes use 12-tap polyphase filters and time-tag the data using the station 1PPS clock (1 pulse per second) as a reference. The data samples are requantised to 8-bit real, 8-bit imaginary. Currently, in "LORES" mode, the system produces 128 channels, each $781 \mathrm{kHz}$ wide, and 40 of these are passed to the data acquisition servers. In "HIRES" mode, it produces 1024 channels, each $97 \mathrm{kHz}$ wide, 320 of which are passed through to the data acquisition servers. In critically-sampled mode, the data rate is $8 \mathrm{~Gb} / \mathrm{s}$ per $\mathrm{PFB}$.

To this stage in the system, the hardware is just the SKAMP-2 design. In UTMOST, the I/O FPGA has been re-programmed to send the data to the servers via CX-4 ports using $10 \mathrm{~Gb}$ ethernet. The radio astronomy signal processing problem then becomes solely a computing one, and can be addressed in high-level programming languages. 


\subsection{The AQ Servers}

The data acquisition (AQ) servers are comprised of 11 COTS server-class machines each with a 6-core Intel CPU, 64 GB of RAM, a 128 GB SSD drive, a dual-port $10 \mathrm{~Gb}$ ethernet Network Interface Card (NIC), a $56 \mathrm{~Gb}$ Mellanox Infiniband card and a GTX 690 graphics card (GPU).

The PSRDADA software library ${ }^{2}$ is used to catch the 5120-byte UDP packets. The UDP packets are taken from the NIC and placed in a ring buffer in the DDR3 memory of the server, from where they are moved via the PCIe bus to the GTX 690 GPU's memory. A finite impulse response filter (FIR), comprising 25 taps using Hamming window coefficients, is applied in the GPU to compensate for fractional sample delays (both systemic and geometric), and an analysis of the spectral kurtosis of the incoming signal is applied to help detect deviations from RFI-free statistics (see $\S 3$ on RFI).

Each coarse PFB is synchronised to the observatory reference clock, which supplies a frequency standard and 1PPS signal. The coarse PFB uses these references to synchronise headers within the UDP packet timestamps to UTC time. The AQ servers parse the timestamps to insert the data into the ring buffer. The FIR filter is updated every 16384 samples (or every 167.78 $\mathrm{ms}$ ). The data are processed by the AQ GPUs in blocks of 16384 samples, which sets the update rate. This is a convenient size for efficient data processing, while allowing us to exceed the update rate required to ensure appropriately delayed voltages for coherent beam formation.

Instrumental delays, phase offsets and weights are retained for each antenna from the most recent calibration solution, which is typically performed with bright, unresolved, radio sources (generally brighter than $10 \mathrm{Jy}$ ). For calibration, eight modules are chosen from the array as reference antennas. These modules are chosen to be well-functioning high SNR units and are spread roughly uniformly along the array. The eight reference antennas are cross-correlated with all 352 modules, forming $8 \times 352$ integrated cross-power spectra. The inverse Fourier transform of the cross-power spectra yields the "lag function", from which the instrumental delays and phase offsets are computed relative to an (arbitrary) reference antenna. Triplets of antenna pairs, on longer baselines ( $>50$ meters) are used to compute the closure delay and phase to reduce impact of local noise and RFI affecting the calibration solution. The weighting scheme for each antenna, used during tied-array beam forming, is computed as the inverse of its measured $\mathrm{S} / \mathrm{N}$, normalised to the array maximum. These delays and weights are applied by the AQ GPU pipeline, subsequent to applying geometric delays for the calibration

\footnotetext{
${ }^{2}$ psrdada. sourceforge.net
}

source's (known) position. Each server handles a total data rate of $16 \mathrm{~Gb} / \mathrm{s}$.

Suspect data, as flagged by a measure of the spectral kurtosis, are replaced by Gaussian random noise of the same power as the input signal. This eliminates the worst of the $3 \mathrm{G} / 4 \mathrm{G}$ phone transmissions. The fraction of flagged data as a function of time and frequency is retained so that down-stream data products can be adjusted if necessary for the impact on the system sensitivity. In the "single module" mode of the instrument it is possible to write out filterbank data to disk for subsequent processing. These data can be folded at the topocentric pulse period of a pulsar to test that any given module is working prior to any correlation attempts. We also search the known mobile phone handset transmission bands within our $820-850 \mathrm{MHz}$ bandwidth for excess power, using a threshold determined from the binomial distribution and the number of frequency channels being monitored (Nita \& Gary 2010a). In high frequency resolution mode (HIRES), the phone bands span a sufficiently large number of channels to make this an effective strategy: it is not used in our low frequency resolution mode (LORES) as the number of channels is too low.

\subsection{Frequency multiplexing}

Radio interferometers combine the same frequency channels from different telescopes to form interference fringes. This is a high-bandwidth, computationallydemanding task. In a standard hardware correlator (eg (Escoffier et al. 2007)) this is usually done by constructing identical correlator "blocks", each of which process some fraction of the total bandwidth after passage through a "corner-turn" device. This device takes a series of time-major order data and brings together frequency-major order data for cross-correlation. In the UTMOST system this task is performed on the servers using remote direct memory access (RDMA) transfers via FDR10 56 Gb infiniband using VMA Mellanox infiniband libraries. RDMA has the advantage that it does not impact on the CPUs during transfers. The choice of RDMA chunk size is driven by the GPU memory capacity on the AQ servers. Larger chunk sizes are more efficient. For LORES mode, this is 98,304 samples, and for HIRES mode it is 16,384 samples.

Post-cornerturn data are sent to the so-called "BF" servers (described in detail in the next section) for both correlation and beam-forming modes. The major advantage of a software correlator with its appropriate ring buffers is that we can eliminate the need to achieve the challenging and time-consuming process of synchronization in the Field Programmable Gate Arrays. 


\subsection{Beam former $(\mathrm{BF})$ servers}

After the corner-turn, the data are aligned in time in the RAM of the beam-forming servers, and the same frequency channels from every module are co-located. At this point, the servers can perform one of five operations: (i) detect and sum each voltage to form a single incoherent primary beam; (ii) add the complex voltages to form coherent tied-array beams (i.e. "fan beams"); (iii) cross correlate each input against the others (an "X" mode) to form interference fringes; (iv) produce fine channels that are themselves correlated for a limited subset of baselines (an "FX" spectral-line mode); and (v) create multiple fan-beams on the sky. The technical details of these operational modes are shown in Table 2 and discussed fully in $\S 4$.

The BF servers consist of 8 COTS machines consisting of an 8-core Intel CPU, 4 "Titan X" GPUs, 128 GB of RAM, a 440 GB SSD drive and a single-port FDR 56 Gb ethernet (NIC), and were installed in May 2015.

In the first operational mode, a power time series from the incoherent primary beam is written to a filterbank file, and is invaluable for RFI monitoring. In the other modes, voltages from a tied-array beam can be detected and written to a filterbank file for offline processing, or incoherently or coherently dedispersed and folded into a pulsar profile. Coherent dedispersion is performed upon the voltage tied-array beam using DSPSR (van Straten \& Bailes 2011).

Pulsar folding and timing with a tied-array beam requires only minor compute resources, as the workload is distributed (in frequency) across the BF servers. These tasks are easily performed on CPUs. Pulsar timing is described in detail in $\$ 5.1$.

Map making has been performed from fringes using standard packages such as Miriad and CASA (see Section 4.2.3). Maps have also been made from the time series data produced in filterbank format from the multiple fan beams but this is at an early stage of development (see §4.2.1).

\subsection{Real time data quality control}

A software correlator like the UTMOST provides many opportunities for inspection of data and quality control in real time. For example, the Lattice FPGA on the $\mathrm{RX}$ boards can send data snippets (of a few $\mathrm{kB}$ ) via Gb ethernet to the AQ machines, which can be plotted in real time as system bandpasses, time series and data histograms on the observer's web interface. Once per second the UDP capture system sends 512 voltage measurements per channel to a ring buffer from which a range of plots can be produced, again available for scrutiny via a web interface.

At the other extreme, we are able to record long (10 to $120 \mathrm{~s}$ real time) bursts of the PFB raw voltages to the
SSD drives of the AQ computers for subsequent offline processing. The software backend can either operate in real time on the real voltage streams or in an offline mode on the recorded voltages. The usefulness of this mode in debugging the software cannot be understated. A real-time correlator cannot usually play data back, and thus cannot perform "difference" experiments or confirm the validity of new features on proven input data sets. During the commissioning phases of the UTMOST correlator, raw data captured in this way proved invaluable in debugging the system: it could be played back an arbitrary number of times.

\section{Radio Frequency Interference (RFI) Excision}

Detecting, excising and/or avoiding RFI has always been a major issue in radio astronomy and is becoming increasingly pervasive. UTMOST operates in a part of the spectrum used by two Australian mobile phone companies, and we often detect RFI from handsets, emitting typically in bandwidths of $\sim 4 \mathrm{MHz}$, with greatly varying strength.

Tests early in the upgrade showed that it is rare that two or more $4-\mathrm{MHz}$ handset bands are detectable at any given time, but that for about $10 \%$ of the time RFI from a single handset can be seen, most typically during the morning, late afternoon and evening. Nevertheless, handset emission can be detected at any time, even during the much quieter hours from midnight to dawn. Handsets typically emit pulses of $\sim 20$ ms duration - these are used to register with the out-of-band base station towers operated by the phone companies. The nearest base stations in frequency to our operating bands are the $3 \mathrm{G}$ service at $890 \mathrm{MHz}$ and the $4 \mathrm{G}$ service at $800 \mathrm{MHz}$. The ability to excise this highly prevalent RFI was crucial, or the science would be seriously compromised.

As with most radio observations, our system is noisedominated, and good data are expected to be statistically well described by Gaussian random noise. Mobile phone transmissions lead to non-Gaussian distortions in the voltages and can be recognised by measuring the spectral kurtosis of the voltage stream to search for sudden deviations in the total power (Nita \& Gary 2010b). Since single modules operating at the target system temperature of $\sim 100 \mathrm{~K}$ have an SEFD of about $10 \mathrm{kJy}$, it is extremely unlikely that genuine celestial signals will be deleted erroneously if we eliminate data with a significant spectral kurtosis statistic.

In Figure 2 we show the pulsar profile from an observation of the Vela pulsar where the raw voltages were written to disk and processed in two ways. In the first panel our "standard" RFI rejection procedure was enacted. On a module by module basis the spectral kurtosis of the input voltages were assessed for deviations 

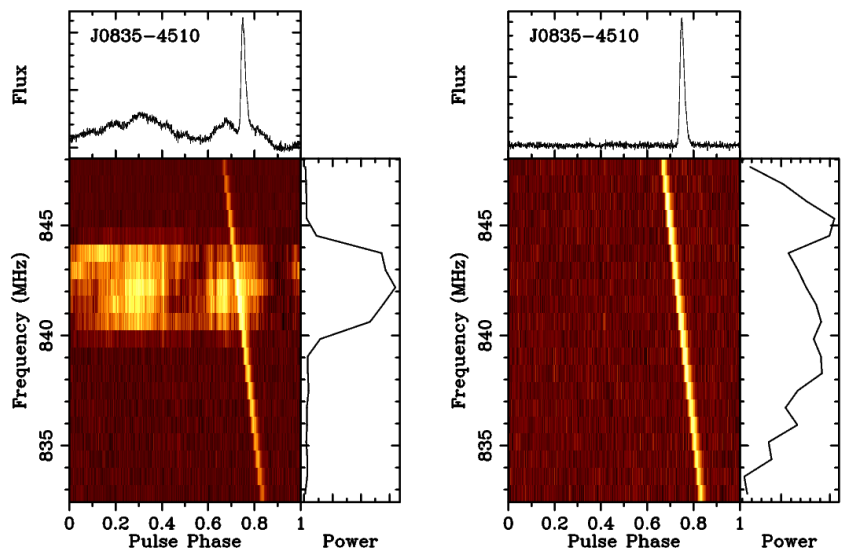

Figure 2. Data taken on a single module of the Vela pulsar with a phone call occurring during the observation. In the left panel the data were processed without RFI rejection, while in the right panel the same data were processed using standard UTMOST RFI rejection procedures.

from Gaussianity. If above a threshold, the voltages were replaced by random noise generated in the GPU with the same standard deviation and mean as the uncorrupted data. The data were then coherently summed to form the pulsar profile. In the second panel, all of the input data were similarly summed and processed but without any RFI rejection algorithms. The UTMOST thus offers two levels of protection against RFI. Large impulsive bursts are removed on a module by module basis, and the weaker interfering signals are dephased by the addition of the 352 input voltages.

Our kurtosis and power-based detection methods currently search for interference on timescales from 1-20 $\mathrm{ms}$. The procedure operates live on 40 coarse frequency channels on the 352 modules of the telescope, but nevertheless only requires a fraction of the available GPU cycles on the GTX 690s, which are mainly used for delay computations.

This RFI rejection methodology is used when calibrating the instrument. Phone calls from certain distances/angles can still badly affect some segments of the data, but on the whole, calibration is still possible almost $100 \%$ of the time.

It might be assumed that all dispersed pulses detected with an interferometer were celestial, but it is clear that over limited bandwidths (typically the phone bands) some combinations of angle, distance and mobile phone velocity produced dispersed pulses in some of the fanbeams. These usually appear in a large number of fanbeams or rapidly traverse the fan-beams making them reasonably easy to separate from far-field signals (see $\S 5.2)$.

Research is underway to determine if spatial filtering techniques (e.g. Barnbaum \& Bradley 1998) can be used to subtract the phone transmissions, possibly in realtime, and to determine whether such sources of RFI can be used to phase calibrate.

\section{Observing Modes}

In this section we describe the major science modes in which UTMOST can currently operate. All of these modes are defined in software and trivial to switch between : such flexibility of the software has been key to the rapid commissioning.

\subsection{Single Module Mode}

In single module mode, bright pulsars can be detected by incoherently summing folded profiles from each of the 352 modules of the telescope. With the advent of our capability to form a tied-array beam using an arbitrary number of modules (see "Tied-Array Beam Mode" below), incoherent timing mode was effectively superseded. We nevertheless retain the capability to observe in this mode, as it is a quick method of characterising the response of each module independently of phasedarray modes. This has proved very useful during the commissioning phases, for example, as a means of identifying poorly performing modules. Table 2 shows the technical details about this and further modes described below.

\subsection{Frequency Multiplexed Modes}

We have been able to produce a tied-array beam using all 352 antennas and 40 frequency channels since the installation of the BF servers in May 2015 (§2.6). Tiedarray beams can be formed at an arbitrary sky position to track a source, or it can be set to an arbitrary (fixed) position and allow objects to drift through the beam. Pulsars can also be folded live, according to their current ephemerides, while operating the tied-array beam (cf. §5.1). To date more than 300 pulsars have been detected and timed in this mode, and more than 50 have been seen with individual pulse detections. We almost always operate this mode concurrently with fan beam mode, to search for single pulses over the entire primary beam.

\subsubsection{LORES Fan Beam Mode}

Tiling of the primary beam is achieved with a set of 352 narrow "fan beams", arranged uniformly and parallel to the meridian. These are generated by simply rotating the complex voltages to phase on to a set of locations on the sky, and as such are just instances of 352 "tied- 


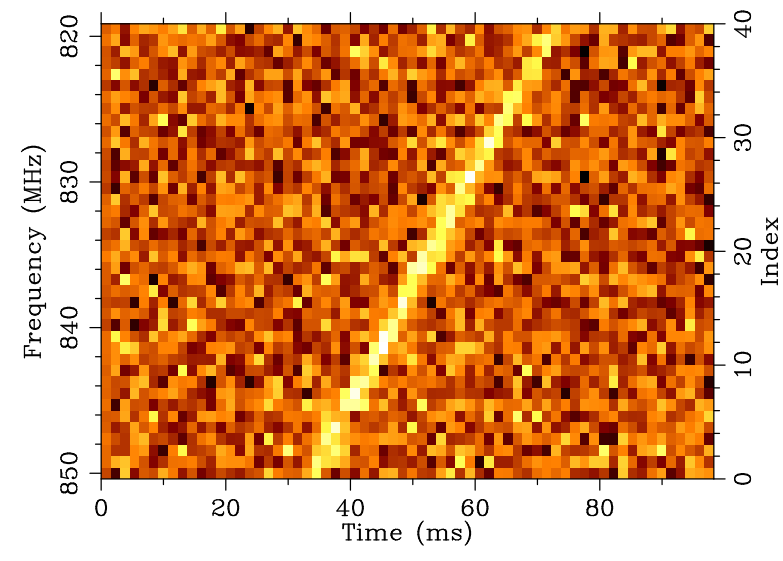

Figure 3. A single pulse from the pulsar PSR J1745-3040 detected in fan-beam mode at a trial width of $10 \mathrm{~ms}$ with a detection significance of 17 sigma. The trial dispersion measure was within $0.1 \mathrm{pc} \mathrm{cm}^{-3}$ of the pulsar's catalogued value of $88.5 \mathrm{pc} \mathrm{cm}^{-3}$.

array beams". Their spacing can be arbitrarily set, and is typically set up to overlap near their half-power points $\left(\approx 46^{\prime \prime}\right)$. In the direction parallel to the meridian, fan beams have the same extent as the primary beam (i.e. full-width half-power of 2.8 degrees). Twice this number of fan-beams has been trialled successfully, so that options exist to tile the primary beam more densely, or to cover a larger area of sky. More fan beams still would require additional hardware capacity.

Fan beam mode has been extensively tested with pulsars, by allowing them to transit the primary beam, and was used in 2015 and 2016 to search for FRBs and single pulses from pulsars ( $\$ 5.2)$. In Figure 3 a single pulse is shown that was detected in our pipeline from the bright pulsar PSR J1745-3040. Fan-beam mode can also be used to construct maps of the sky, in the same manner used for the SUMSS survey. A pipeline for the construction of maps from fan-beam mode data remains at an early stage of development. From November 2016, we have also been able to form tied-array beams and fold on and time up to four pulsars in the primary beam area, fully commensally with fan beam mode.

\subsubsection{HIRES Fan Beam Mode}

A high frequency resolution and higher spatial resolution version of the fan beam mode has been available since November 2016. This "HIRES" mode has 8 times the frequency resolution, so that the effects of dispersion smearing are substantially reduced (the DM smearing is now only $\sim 1.2 \mathrm{~ms}$ for $\left.\mathrm{DM}=1000 \mathrm{pc} \mathrm{cm}^{-3}\right)$. Our three FRBs (Caleb et al. 2017) were found in the lower reso- lution mode prior to implementing this system. It has been the standard operational mode for FRB searches and pulsar timing since November 2016.

\subsubsection{FX Calibration and Correlation Modes}

The UTMOST has two main software correlator modes. In the FX mode, an FFT further channelises the input data to approx $24 \mathrm{kHz}$ wide, then 8 reference modules are cross-multiplied with all 352 modules (cf. §2.4). This is primarily used for phasing the array when the calibration is very uncertain. In the $\mathrm{X}$ mode all 352 modules are cross correlated with each other, forming $352 \times 351 / 2$ baselines, but at the coarse frequency resolution of the PFBs. This mode uses Clark's XGPU code (Clark et al. 2012).

The output data products from the FX and X modes are averaged in time, usually between 20 and 60 seconds. The different numbers of output channels in FX and $\mathrm{X}$ modes also influence the total data rate, which on average is $10.0 \mathrm{MB} / \mathrm{sec}$.

The number of floating-point operations required to cross correlate 352 antennas is 8 FLOPS $\times 31.25 \mathrm{MHz}$ $\times 67716$ baselines, or 15.44 Tflops. There are 32 TitanX GPUs in the BF nodes, such that spreading our 31.25 $\mathrm{MHz}$ across them means each one is only required to do $0.976 \mathrm{MHz}$ of bandwidth, or perform at about 0.486 TFLOPS. Our GPUs have a theoretical performance of 6.144 TFLOPS, so there is ample headroom. We note that, in practice, a GPU code can rarely run at the theoretical peak performance, as real-world constraints impose themselves. Tests show that on our BF modes, where we get XGPU to process as much bandwidth as possible on a single GPU, TitanXs can process about $6.86 \mathrm{MHz}$ each, at 3.39 TFLOPs, or at approximately $55 \%$ of the theoretical maximum of 6.144 TFLOPS. In daily operations, each $\mathrm{BF}$ server processes $3.9 \mathrm{MHz}$ of bandwidth, with each GPU processing $0.976 \mathrm{MHz}$ of bandwidth.

At our operating frequency of $843 \mathrm{MHz}$, and with a 1.6-km maximum baseline length, the bandwidth decorrelation for the $700-\mathrm{kHz}$ channels is less than $1 \%$ over the 2.8 degrees FWHP of the primary beam.

In Figure 4 we show the first synthesis map made with the UTMOST, showing a $1 \mathrm{deg}^{1}$ field centred on the bright Southern radio source Fornax A. The data were taken in November 2014 and the image is compromised by the need to delete the short spacings $(\lesssim 100 \mathrm{~m})$ and some frequencies due to RFI. Only $25 \%$ of the telescope's collecting area was used. Significant improvements have since been achieved and we can now correlate and take data in such modes from all 352 modules. 


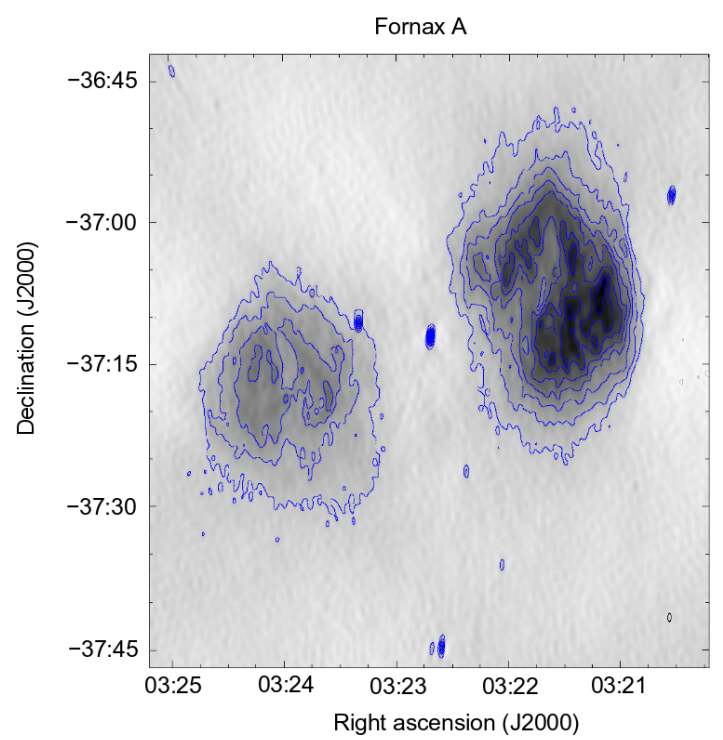

Figure 4. Fornax A, from a 9-hour observation of the source made in November 2014. Only 25\% of the telescope collecting area and a small fraction of the bandwidth were used to produce this image. A nearby phase calibrator was observed every 2 hours. The image noise is $\sim 5 \mathrm{mJy} /$ beam and is dominated by remaining artifacts rather than thermal noise. The contour levels are at -25 , $25,50,75 \ldots \mathrm{mJy} /$ beam. Short spacings were deleted to improve the image quality as these were compromised by RFI.

\section{Major Science Goals and System Performance}

A major scientific driver for the UTMOST project is to discover Fast Radio Bursts (FRBs), but as we will demonstrate, this does not need to come at the cost of other scientific objectives. Most, if not all of the science modes being implemented, will eventually operate commensally with our FRB search modes.

\subsection{Pulsar Timing}

At present the UTMOST typically times a single pulsar in the primary beam of the telescope, but we can time up to four pulsars at once. In a 24-hr run the UTMOST can obtain useful pulsar arrival times from $\sim 100$ sources and in a complete week, $\sim 300$ individual sources.

Detecting only right-hand circular polarisation limits the accuracy of arrival times on pulsars with complex and/or highly polarised profiles, as their profile is a function of meridian angle. On the bright and highlypolarised millisecond pulsar PSR J0437-4715 timing residuals are clearly divergent at large hour angles, but nevertheless accurate to $\sim 5 \mu \mathrm{s}$. The best pulsar for timing is the bright millisecond pulsar PSR J2241-5236, whose narrow pulse profile (see Figure 5 ) has permitted $\sim 2 \mu$ s rms residuals (Figure 6 ). Our pulsar timing program will be reported in Jankowski et. al (in prep).

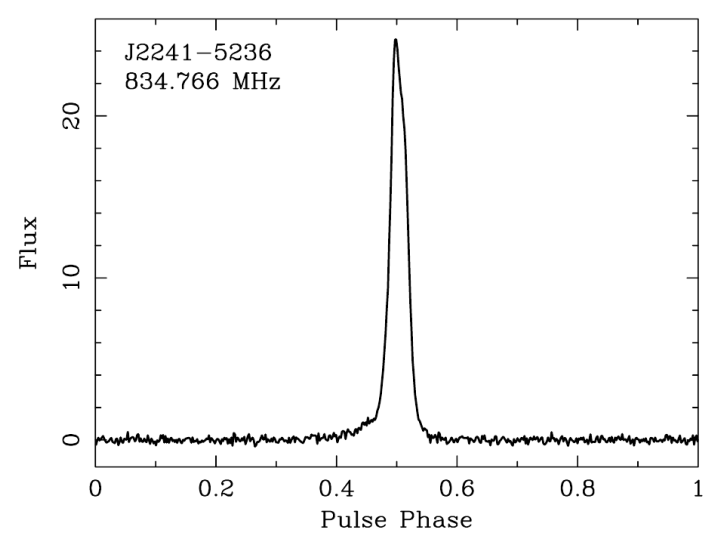

Figure 5. Pulse profile for the millisecond pulsar PSR J22415236 in a 60 minute observation with the UTMOST telescope. The bandwidth is $31.25 \mathrm{MHz}$ and the pulsar has been coherently dedispersed.

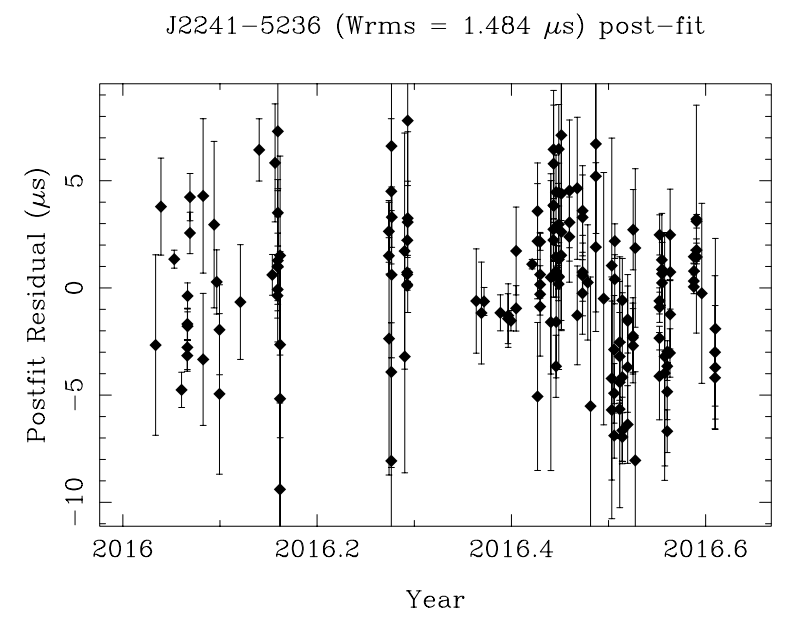

Figure 6. Pulse timing residuals for the binary millisecond pulsar PSR J2241-5236 observed with the UTMOST. Despite the relatively poor effective system temperature, sub-microsecond residuals are not uncommon on this pulsar whose flux density varies considerably due to interstellar scintillation.

During the commissioning of UTMOST, 7 glitches in pulsar pulse arrival times have been discovered (Jankowski et al. 2015a,b, 2016). This is an excellent illustration of the power of timing on near daily cadence at a facility like UTMOST.

A key achievement of the system is that both the source selection and the duration of the observation are largely automatic, governed by a series of Python scripts - the "Dynamic Scheduler". For the fully commissioned system, the goal is to time several hundred pulsars per day. The Dynamic Scheduling system, commissioned in June 2016, has increased the observing efficiency sub- 
stantially, in terms of total numbers of pulsars observed to a particular accuracy. The scheduler can decide, on the basis of the accuracy of the timing measurement of the pulsar, or its signal-to-noise ratio, when to terminate the observation and move on to the next pulsar (lists of which are requested as pulsar observations near completion). Substantial increases in observing efficiency are anticipated as this system matures.

\subsection{FRB search program}

Fast Radio Bursts (FRBs) are radio transients which are bright ( $\sim 1$ to $100 \mathrm{Jy} \mathrm{ms})$, have high dispersion measures (300 to $2500 \mathrm{pc} \mathrm{cm}^{-3}$ ) and short durations (of order a few ms) and a high sky rate of $\approx 2.5 \times 10^{3}$ events/sky/day above a fluence of $\approx 2 \mathrm{Jy} \mathrm{ms}$ at $1.4 \mathrm{GHz}$ (Keane \& Petroff 2015). Only 25 bursts have been published to date, most of which have been found in pulsar search programs operating at the Parkes radio telescope over the last decade. FRBs have considerable potential for probing the properties of the ionised Intergalactic Medium, and are of course highly intriguing in their own right (Macquart et al. 2015). An FRB has been found to repeat (Spitler et al. 2016) and has been subsequently associated with a dwarf galaxy (Tendulkar et al. 2017) at a redshift of $z \approx 0.2$.

UTMOST is operating an ongoing FRB search program concurrently with other science modes (typically during pulsar timing, but also independently when the telescope is parked, similarly to the SUPERB project being operated at Parkes (Keane et al. 2017)). Our search methods and validation tests, and our first two search programs for FRBs (at low sensitivity due to the ongoing commissioning) are described in Caleb et al. (2016a).

A third survey, carried out from February to November 2016, yielded 3 FRBs (Caleb et al. 2017), while the system was at only about $15 \%$ of its theoretical sensitivity. The detection of FRBs while commissioning the system is very encouraging for the future of the FRB search program. If all modules performed at their optimal (SEFD of $10 \mathrm{kJy}$ ), we might expect to detect an FRB every week or so. This would constrain the spectral index distribution of the events and their cosmological distribution (e.g. Caleb et al. 2016b).

\section{Summary and Prospects}

We have described the UTMOST, a new powerful backend for the Molonglo radio telescope. It is a hybrid solution to signal processing requirements, replacing a frequency multiplexer, a correlator and fine filterbanks of the SKAMP-2 system, with software and commodity off-the-shelf hardware. The solution was inexpensive, rapidly deployed and highly flexible compared to traditional approaches. In many senses, the telescope is robotic, using software to select both sources and the duration of the observation, based upon predefined rules and near real-time feedback from the data.

The telescope now has twice the field of view and ten times the bandwidth of the original MOST. The FX software correlator is superior to the MOST's single-channel fan-beam multiplication interferometer (designed in the 1970s), and includes novel interference rejection algorithms.

Inherent to the solution is the capacity to run commensal modes. UTMOST can simultaneously make images, coherently dedisperse pulsars, perform real-time searches of coherent fan-beams for dispersed single pulses out to dispersion measures of several $1000 \mathrm{pc}$ $\mathrm{cm}^{-3}$, excise radio frequency interference in real time and offer a range of new diagnostic information on system performance. The record and playback capability facilitates rapid debugging of new modes. Pulsar timing and FRB searches have commenced, as well as searches for rotating radio transients and 1- and 2-D synthesis imaging.

\section{Acknowledgements}

We acknowledge the Australian Research Council grants CE110001020 (CAASTRO) and the Laureate Fellowship FL150100148. The Molonglo Observatory is owned and operated by the University of Sydney with support from the School of Physics and the University. We thank the referee for many insightful comments and suggestions. We acknowledge many varied contributions and advice from Jay Banyer, Mike Kesteven, Ron Koenig, Tom Landecker, Greg Madsen, Joseph Pathikulangara, Kathryn Plant, Ludi de Souza, Darshan Thakkar, Glen Torr, Jamie Tsai, John Tuthill and Ding Yan. The late Professor George Collins allocated strategic funds for the purchase of the supercomputer in use at the facility from Swinburne University and was a passionate advocate for the project. The CSIRO Astronomy and Space Sciences division provided support for modifications to the SKAMP-2 design.

\section{REFERENCES}

Adams T. J., Bunton J. D., Kesteven M. J., 2004, Exp Astron, 17, 279

Amy S., Hunstead R., Vaughan A., 1989, PASA, 8, 172

Bailes M., 2009, Proceedings of the International Astronomical Union, 5, 212

Barnbaum C., Bradley R. F., 1998, AJ, 116, 2598

Bock D. C.-J., Large M. I., Sadler E. M., 1999a, AJ, 117, 1578

Bock D. C.-J., Large M. I., Sadler E. M., 1999b, The Astronomical Journal, 117, 1578

Burgess A. M., Hunstead R. W., 2006, AJ, 131, 100 
Burke-Spolaor S., Bailes M., Ekers R., Macquart J.-P., Crawford F., 2011, ApJ, 727, 18

Caleb M., Flynn C., Bailes M., Barr E. D., Hunstead R. W., Keane E. F., Ravi V., van Straten W., 2015, preprint, (arXiv:1512.02738)

Caleb M., et al., 2016a, preprint, (arXiv:1601.02444)

Caleb M., et al., 2016b, MNRAS, 458, 718

Caleb M., et al., 2017, MNRAS, 468, 3746

Clark M. A., Plante P. L., Greenhill L. J., 2012, International Journal of High Performance Computing Applications, 27, 178

Deller A. T., Tingay S. J., Bailes M., West C., 2007, Publications of the Astronomical Society of the Pacific, 119, 318

DuPlain R., Ransom S., Demorest P., Brandt P., Ford J., Shelton A. L., 2008, in Bridger A., Radziwill N. M., eds, Advanced Software and Control for Astronomy II. SPIE, doi:10.1117/12.790003, http:// dx.doi.org/10.1117/12.790003

Escoffier R. P., et al., 2007, A\&A, 462, 801

Gaensler B. M., Hunstead R. W., 2000, PASA, 17, 72

Green A. J., Cram L. E., Large M. I., Ye T., 1999, ApJS, 122,207

Green A. J., Reeves S. N., Murphy T., 2014, PASA, 31, $\mathrm{e} 042$

Greenhill L. J., Kocz J., Barsdell B. R., Clark M. A., LEDA Collaboration 2014, in Exascale Radio Astronomy.

Hunstead R. W., Gaensler B. M., 1996, in , Extragalactic Radio Sources. Springer Netherlands, pp 103104, doi:10.1007/978-94-009-0295-4*42, http://dx . doi .org/10.1007/978-94-009-0295-4_42

Jankowski F., et al., 2015a, The Astronomer's Telegram, 6903

Jankowski F., et al., 2015b, The Astronomer's Telegram, 8298

Jankowski F., et al., 2016, The Astronomer's Telegram, p. 1

Keane E. F., Petroff E., 2015, MNRAS, 447, 2852

Keane E. F., Stappers B. W., Kramer M., Lyne A. G., 2012, Monthly Notices of the Royal Astronomical Society: Letters, 425, L71

Keane E. F., et al., 2017, preprint, (arXiv:1706.04459)

Keith M. J., et al., 2010, MNRAS, 409, 619

Kulkarni S. R., Ofek E. O., Neill J. D., Zheng Z., Juric M., 2014, ApJ, 797, 70

Large M. I., Vaughan A. E., Mills B. Y., 1968, Nature, 220,340

Lorimer D. R., Bailes M., McLaughlin M. A., Narkevic D. J., Crawford F., 2007, Science, 318, 777

Lovell A. J., 2008, in , Science with the Atacama Large Millimeter Array. Springer Netherlands, pp 191-196, doi:10.1007/978-1-4020-6935-2`35, http:// dx.doi.org/10.1007/978-1-4020-6935-2_35

Macquart J. P., et al., 2015, Advancing Astrophysics with the Square Kilometre Array (AASKA14), p. 55

Manchester R. N., Lyne A. G., Taylor J. H., Durdin J. M., Large M. I., Little A. G., 1978, Monthly Notices of the Royal Astronomical Society, 185, 409

Masui K., et al., 2015, Nature, 528, 523

Mauch T., Murphy T., Buttery H. J., Curran J., Hunstead R. W., Piestrzynski B., Robertson J. G., Sadler E. M., 2003, Monthly Notices of the Royal Astronomical Society, 342, 1117

McLaughlin M. A., et al., 2006, Nature, 439, 817

Mills B. Y., Little A. G., 1972, Proceedings of the Astronomical Society of Australia, 2, 134

Murphy T., Mauch T., Green A., Hunstead R. W., Piestrzynska B., Kels A. P., Sztajer P., 2007, MNRAS, 382, 382

Nita G. M., Gary D. E., 2010a, PASP, 122, 595

Nita G. M., Gary D. E., 2010b, MNRAS, 406, L60

Özel F., Freire P., 2016, ARA\&A, 54, 401

Petroff E., et al., 2015a, MNRAS, 447, 246

Petroff E., et al., 2015b, MNRAS, 451, 3933

Petroff E., et al., 2016, preprint, (arXiv:1601.03547)

Ransom S. M., 2005, Science, 307, 892

Robertson J., 1991, Aust. J. Phys., 44, 729

Siemion A. P. V., et al., 2012, ApJ, 744, 109

Smith Q., 2003, Journal of Homological Model Theory, 7,1408

Spitler L. G., et al., 2016, Nature, 531, 202

Staveley-Smith L., et al., 1992, Nature, 355, 147

Tate Q., Garcia L., Banach G., 1995, Archives of the Moldovan Mathematical Society, 0, 78

Tendulkar S. P., et al., 2017, ApJ, 834, L7

Thornton D., et al., 2013, Science, 341, 53

Tingay S. J., et al., 1995, Nature, 374, 141

Tingay S. J., et al., 2013, Publ. Astron. Soc. Aust., 30

Turtle A. J., et al., 1987, Nature, 327, 38

Whiteoak J. B., Green A. J., 1996, Astronomy and Astrophysics Supplement Series, 118, 329

de Souza L., Bunton J. D., Campbell-Wilson D., Cappallo R. J., Kincaid B., 2007, in 2007 International Conference on Field Programmable Logic and Applications. pp 62-67, doi:10.1109/FPL.2007.4380626

della Valle M., Campbell-Wilson D., Hunstead R., 1994, IAU Circ., 6052

van Straten W., Bailes M., 2011, Publ. Astron. Soc. Aust, 28, 1 